\title{
Changes in the gene mutation profiles of circulating tumor DNA detected using CAPP-Seq in neoadjuvant chemotherapy-treated advanced ovarian cancer
}

\author{
TOMOKO NOGUCHI $^{1}$, KAZUKO SAKAI $^{2}$, NAOYUKI IWAHASHI ${ }^{1}$, KAHO MATSUDA $^{1}$, \\ HITOMI MATSUKAWA $^{1}$, TAMAKI YAHATA ${ }^{1}$, SAORI TOUJIMA ${ }^{1}$, KAZUTO NISHIO $^{2}$ and KAZUHIKO INO ${ }^{1}$ \\ ${ }^{1}$ Department of Obstetrics and Gynecology, Wakayama Medical University, Wakayama, Wakayama 641-0012; \\ ${ }^{2}$ Department of Genome Biology, Kindai University Faculty of Medicine, Osaka-Sayama, Osaka 589-8511, Japan
}

Received October 31, 2019; Accepted December 3, 2019

DOI: $10.3892 / 01.2020 .11356$

\begin{abstract}
Cancer Personalized Profiling by deep Sequencing (CAPP-Seq) is a novel ultrasensitive next-generation sequencing-based approach that is used to detect circulating tumor DNA (ctDNA). The aim of the present study was to compare the gene mutation profiles and blood tumor mutation burden (bTMB) measured between pre- and post-neoadjuvant chemotherapy (NAC), utilizing CAPP-seq for plasma ctDNA in patients with advanced ovarian cancer. The current study included 10 patients (6 NAC-sensitive and 4 NAC-resistant) clinically diagnosed as having stage III or IV ovarian cancer and were administered NAC between May 2017 and February 2019. The plasma ctDNA samples were collected at pre- and post-NAC, and comprehensive gene mutation analysis was performed using CAPP-seq. In 5 out of 6 NAC-sensitive cases, the variant allele frequency (VAF) of non-synonymous somatic mutations decreased following NAC. In 2 out of the 4 NAC-resistant cases, the VAF of non-synonymous somatic mutations increased, and new somatic mutations emerged following NAC. In regard to TP53 mutation, the rate of TP53 mutation in the NAC-resistant cases was significantly higher compared with NAC-sensitive cases. Finally, the bTMB decreased significantly after NAC treatment in the NAC-sensitive cases, even though there were no significant differences in the pretreatment bTMB levels between the NAC-sensitive and NAC-resistant cases. These results indicated that gene mutation can be profiled and monitored using liquid biopsy-based CAPP-Seq in patients with advanced ovarian cancer with NAC treatment, and TP53 mutation in the
\end{abstract}

Correspondence to: Professor Kazuto Nishio, Department of Genome Biology, Kindai University Faculty of Medicine, 377-2 Ohno-higashi, Osaka-Sayama, Osaka 589-8511, Japan

E-mail:knishio@med.kindai.ac.jp

Key words: ovarian cancer, CAncer Personalized Profiling by deep sequencing, liquid biopsy, neoadjuvant chemotherapy, circulating tumor DNA
ctDNA and bTMB may be novel biomarkers that can be used for patient monitoring during NAC treatment.

\section{Introduction}

Ovarian cancer is the cancer that carries the worst prognosis among gynecological malignancies (1), and the majority of cases are diagnosed at an advanced stage with peritoneal metastases. The standard therapy for advanced ovarian cancer is primary debulking surgery (PDS) followed by chemotherapy, and complete/optimal primary cytoreduction can improve the survival (2). However, in cases where incomplete surgery is anticipated, neoadjuvant chemotherapy (NAC) followed by interval debulking surgery (IDS) is recommended as a treatment option (3-5). Although platinum/taxane-based chemotherapy is usually chosen as a 1st line regimen for NAC, a significant number of patients show resistance to NAC. Furthermore, it is a disadvantage that sufficient tumor samples may not be acquired by cytology or laparoscopic biopsy before the start of NAC. Some studies have indicated that change in the serum levels of cancer antigen 125 (CA125) could serve as a predictor of monitoring the response to NAC (6-8), however its usefulness is often limited because no significant elevation of any specific serum tumor marker is observed in some histological types of ovarian cancer. Thus, novel and reliable molecular biomarkers related more closely to the intrinsic molecular/biological characteristics of independent ovarian cancers are needed, rather than the conventional serum tumor markers to monitor the treatment response and predict the efficiency of NAC.

Liquid biopsies of circulating tumor DNA (ctDNA) released into the plasma from necrotic and apoptotic cancer tissues have recently been used as a non-invasive diagnostic tool for detecting tumor-specific gene mutations. Liquid biopsy has advantages over tumor biopsy in that it is minimally invasive and the samples can be analyzed in real-time and repeatedly. Furthermore, the molecular characteristics reflected by liquid biopsy may more closely represent tumor heterogeneity compared to those reflected by tumor biopsy (9). Recently, digital PCR $(10,11)$ and next-generation sequencing (NGS) $(12,13)$ have enabled molecular profiling 
of plasma ctDNA. Digital PCR-based methods have very high analytical sensitivity for minor alleles $(\sim 0.01 \%)$ with improved specificity, but in general, can only interrogate one or a few genomic positions simultaneously $(12,13)$. As a tool that allows these limitations to be overcome, Cancer Personalized Profiling by deep sequencing (CAPP-Seq) has recently been developed as the first NGS-based ctDNA analysis method that allows both an ultralow detection limit and broad patient coverage, allowing for a lower amount of input DNA and a lower sequencing cost $(14,15)$. CAPP-Seq shows similar sensitivity for hotspot alleles as digital PCR, and can simultaneously interrogate thousands of additional genomic positions without its sensitivity or specificity being affected. This ultrasensitive technique can detect plasma ctDNA in patients with early and advanced stages of various human malignancies, including lung cancer, lymphoma, and leiomyosarcoma (15-17). We first reported the feasibility of CAPP-Seq-based liquid biopsy in gynecological cancers including ovarian cancer, cervical cancer, and endometrial cancer $(18,19)$. Otsubo et al reported using liquid biopsy-based CAPP-Seq, that the T790M mutation of $E G F R$ is associated with amplification of $M E T$, $E R B B 2$, or $E G F R$ in non-small cell lung cancer (NSCLC) patients resistant to EGFR-TKIs (20). However, there have been no reports about gene mutation profiles using CAPP-Seq for plasma ctDNA obtained from advanced ovarian cancer patients receiving NAC.

In addition, recent studies have shown that the tumor mutation burden (TMB) as determined by targeted NGS might be associated with the response to immunotherapy in patients with lung cancer (21). Furthermore, tissue-based TMB (tTMB) has been reported to be positively correlated with blood-TMB (bTMB) (22), suggesting bTMB could be a surrogate marker of TMB. Gandara et al demonstrated that the bTMB might be associated with the response to immune checkpoint inhibitor therapy in patients with non-small lung cancer (22). It has been reported that CAPP-Seq may be potentially useful as a technique for measuring the bTMB in early/-advanced cancer patients and for monitoring the ctDNA during treatment $(14,15)$. In the gynecologic oncology field, it has been reported that in patients with high grade serous ovarian cancer, the TMB is associated with the treatment response and survival (23), although there have been no reports on the usefulness of the bTMB in patients with gynecologic cancer.

In the present study, we applied CAPP-seq, a ctDNA-based form of targeted NGS, to compare the variant allele frequency (VAF) of tumor-derived somatic mutations in ctDNA measured pre- and post-NAC in plasma samples obtained from ovarian cancer patients receiving NAC. We also examined the changes of the bTMB during NAC treatment as a potential novel biomarker.

\section{Materials and methods}

Patients and samples. This study was conducted in 10 patients who were diagnosed as having stage III or IV ovarian cancer and received NAC between May 2017 and February 2019 at Wakayama Medical University Hospital. The initial diagnosis was based on the clinical findings, including the findings of imaging [computed tomography (CT), magnetic resonance imaging (MRI), and positron emission tomography/computed tomography (PET/CT)] and the cytology/histology of ascitic and pleural fluids. The regimen used for NAC was paclitaxel $\left(175 \mathrm{mg} / \mathrm{m}^{2}\right.$, day 1 ) and carboplatin (5 areas under the curve, day 1) with or without of bevacizumab (15 mg/ $\mathrm{kg}$, day 1), every 21 days. Patient no. 1 developed allergy to paclitaxel during the first course, and docetaxel $\left(60 \mathrm{mg} / \mathrm{m}^{2}\right)$ with carboplatin was administered during the $2 \mathrm{nd} / 3 \mathrm{rd}$ course. Patient no. 8 received weekly paclitaxel $\left(80 \mathrm{mg} / \mathrm{m}^{2}\right.$, day $\left.1 / 8 / 15 / 22\right)$ and bevacizumab $(10 \mathrm{mg} / \mathrm{kg}$, day $1 / 15)$ because of renal dysfunction. Patient no. 9 showed resistance to paclitaxel and carboplatin, and the regimen was changed to cisplatin $\left(60 \mathrm{mg} / \mathrm{m}^{2}\right.$, day 1) plus irinotecan $\left(60 \mathrm{mg} / \mathrm{m}^{2}\right.$, day $\left.1 / 8 / 15\right)$. The number of cycles of NAC was determined based on the clinical treatment response including the clinical findings and serum levels of tumor markers, at the discretion of the treating physician. Nine patients received IDS following NAC. Patient no. 9 did not receive IDS because of her poor performance status. The postoperative pathological diagnosis was determined based on the findings of the resected tumors at the time of IDS.

Blood samples were obtained pre- and post-NAC. The response to chemotherapy was assessed using Response Evaluation Criteria in Solid Tumors (RECIST) version 1.1 (24). In this study, patients with complete response (CR) and partial response (PR) were defined as NAC-sensitive, while those with stable disease (SD) and progressive disease (PD) were defined as NAC-resistant. This study was conducted with approval of the Ethics Committee of Wakayama Medical University (authorization no. 2025) and Kindai University Faculty of Medicine (authorization no. 29-066). Written informed consent was obtained from each of the patients.

Circulating tumor DNA extraction. Peripheral blood samples $(8.5 \mathrm{ml})$ were collected from the patients in cell-free DNA collection tubes (Roche Diagnostics). Plasma ctDNA was purified using an AVENIO cfDNA isolation kit (Roche Diagnostics), in accordance with the manufacturer's instructions. The quality and quantity of the DNA was verified using the PicoGreen dsDNA assay kit (Life Technologies; Thermo Fisher Scientific, Inc.). The extracted ctDNA was stored at $-80^{\circ} \mathrm{C}$ until the analysis.

Circulating tumor DNA sequencing. The CAPP-Seq of ctDNA (10-50 ng) was performed using the AVENIO ctDNA surveillance kit (Roche Diagnostics, 197 genes) as recently described (18-20). The purified libraries were pooled and sequenced on an Illumina NextSeq 500 (Illumina) using the 300-cycle high output kit. Variants were called with the AVENIO ctDNA Analysis Software (Roche Diagnostics), which includes bioinformatics methods from CAPP-Seq (14) and integrated digital error suppression (15). Genetic variants previously cataloged by the Exome Aggregation Consortium at a frequency of $\geq 1 \%$ were excluded, and only non-synonymous single nucleotide variants (SNVs), insertions-deletions (Indels), copy number variations (CNVs), and gene fusions involving 197 cancer-related genes were extracted. Twenty plasma samples obtained from 10 patients treated with NAC were successfully analyzed by CAPP-Seq. The bTMB in each sample was evaluated as the number of non-synonymous mutations number per $\mathrm{Mb}$. 
Table I. Clinico-pathological characteristics of 10 NAC-treated patients with ovarian cancer.

\begin{tabular}{cccccc}
\hline Case no. & Age & Stage & $\begin{array}{c}\text { Postoperative histological } \\
\text { diagnosis }\end{array}$ & Clinical response to \\
NAC
\end{tabular}

NAC, neoadjuvant chemotherapy; HGSC, High-grade serous carcinoma; TC, carboplatin + paclitaxel; DC, carboplatin + docetaxel; Bev, Bevacizumab; CDDP, cisplatin; CPT-11, Irinotecan; PR, partial response; CR, complete response; PD, progressive disease.

Statistical analysis. Statistical analyses were performed using the JMP Pro statistical software version 13.1.1 for Windows (SAS Institute Inc.). Statistical comparisons between the groups were performed using the Mann-Whitney U test. Differences were considered to be significant at $\mathrm{P}$-values of $<0.05$.

\section{Results}

Patient characteristics. The clinicopathological characteristics and response to NAC of the 10 patients are summarized in Table I. The median age of the patients was 63.5 years (44-74 years). Of the 10 patients, six (patient no. 1-6) were NAC-sensitive, and four (patient no. 7-10) were NAC-resistant. The postoperative histological diagnosis in all the NAC-sensitive patients was high grade serous carcinoma. In the NAC-resistant patient group, 2 cases had high-grade serous carcinoma, and one case was mucinous carcinoma. We could not diagnose the histological type in patient no. 9 because she did not receive IDS on account of her poor performance status.

Association of the ctDNA concentration with the response to chemotherapy. The concentrations of ctDNA in plasma samples collected pre- and post-NAC were measured by fluorometry (Fig. S1). The median ctDNA concentrations in the pre- and post-NAC samples of NAC-sensitive patients were 2,335 and 2,198 copies/ml, respectively. On the other hand, in the NAC-resistant cases, the median ctDNA concentrations in the pre- and post-NAC samples were 3,558 and 5,155 copies $/ \mathrm{ml}$, respectively. There were no significant differences in the baseline ctDNA concentration between the NAC-sensitive and NAC-resistant patients. There were no significant changes in the ctDNA concentration between the pre- and post-NAC samples in either NAC-sensitive or NAC-resistant patients.

Mutation status in pre-and post-NAC ctDNA samples. The non-synonymous somatic mutations detected by CAPP-Seq are shown in the NAC-sensitive and NAC-resistant patients in ctDNA obtained pre-NAC (Fig. 1A) and post-NAC (Fig. 1B). Various types of non-synonymous mutations were detected in all the ctDNA samples, however, TP53 mutations were the most frequently detected $(6 / 10,60 \%)$. It is noteworthy that EGFR amplification was detected in the post-NAC sample from the NAC-resistant group (patient no. 8 and 10). Some of the mutations such as NYAP2, SLITRK5, RET, GRM1, FAT1, LRRTM1, BRINP2, CDH9 and GRM1 were detected only in the post NAC samples, like EGFR amplification. These mutations, especially LRRTM1, BRINP2, CDH9 and GRM1 might be related to acquired resistance to NAC, however, it still remains unclear whether these mutations are actionable or not.

On the other hand, mutations of several genes were detected in the pre-NAC but not post-NAC samples: $E G F R$, APC, NRAS, C6orf118, MAP7D3, BRINP2, NAV3, ZNF521, FAM71B, PGK2, ZFPM2, NPAP1, SLC39A12 and MET. Interestingly, EGFR mutation (p.L833F) detected in pre-NAC ctDNA in one case (patient no. 6), but its mutation disappeared in post NAC ctDNA. EGFR mutation is often detected in non-small cell lung cancer but is rare in ovarian cancers. Therefore, the detection of the EGFR mutations in ovarian cancer is a noteworthy finding, although it remains unclear whether this mutation is pathogenic (actionable) or not.

Change in the variant allele frequency (VAF) during NAC. Next, we focused on the changes in the VAFs of TP53 mutation and other somatic mutations, which could be monitored in ctDNA both at pre- and post-NAC (Figs. 2 and 3). Five cases from the NAC-sensitive patient group were monitored (Fig. 2). In patient no. 1, 2 and 3,TP53 mutations were detected in the pre-NAC samples but not in the post-NAC samples. In patient no. 2, the VAF of KCAN5 mutations decreased slightly during the treatment. The VAF of TP53 in patient no. 4 and the GJA8 mutation in patient no. 6 decreased during treatment.

Two patients from the NAC-resistant group (patient no. 7 and 10) could be monitored (Fig. 3). In Patient no. 7, KRAS mutation was detected in the pre-NAC ctDNA but three other kinds of TP53 mutations as well as KRAS mutations were detected in the post-NAC ctDNA. In patient no. 10, VAFs of SLC39A12 mutation was not detected, but VAFs of TP53 and TRPS1 mutations increased after the NAC. These results 


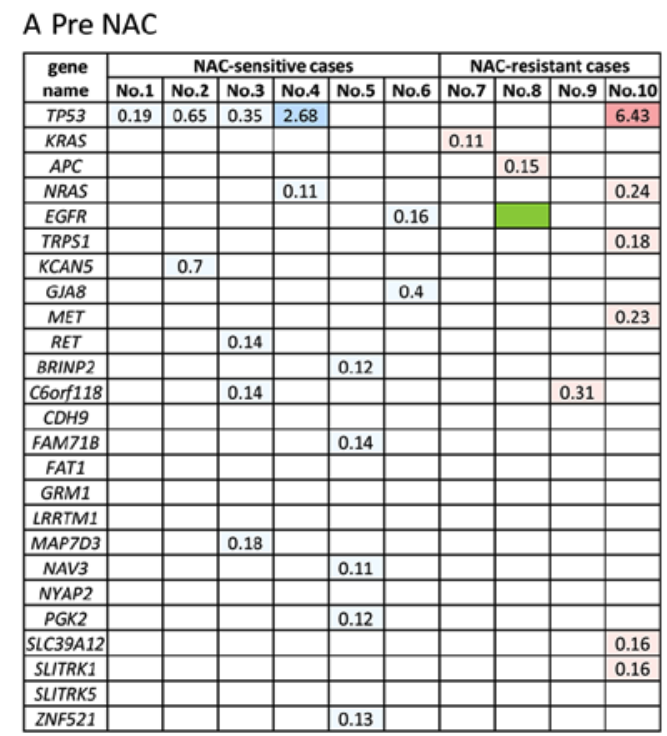

\section{B Post NAC}

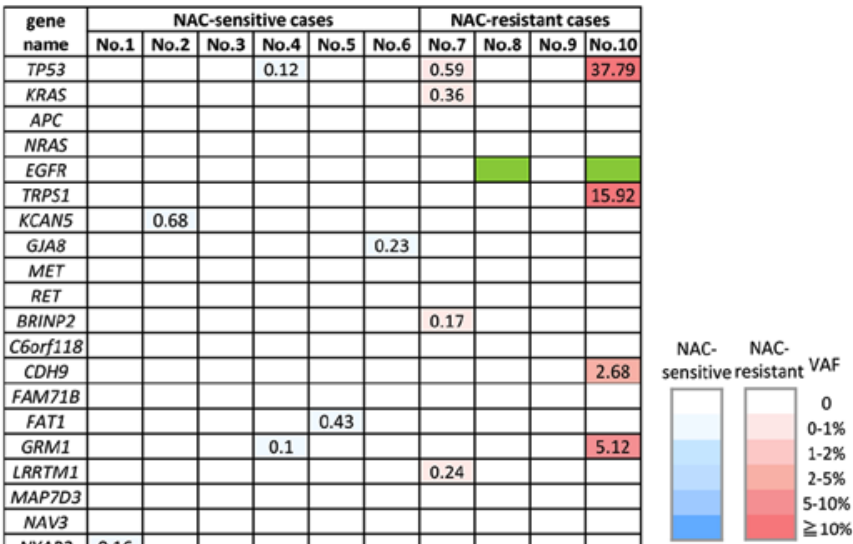

Copy Number Gain (CNG)

Figure 1. Profiling of non-synonymous somatic mutations and amplification detected in circulating tumor DNA obtained (A) pre-NAC and (B) post-NAC. The number in the box indicated VAF of gene mutations. Blue, non-synonymous somatic mutations in NAC-sensitive patient groups; Red, non-synonymous somatic mutations in NAC-sensitive patient groups; Green, copy number gain. NAC, neoadjuvant chemotherapy; VAF, variant allele frequency.
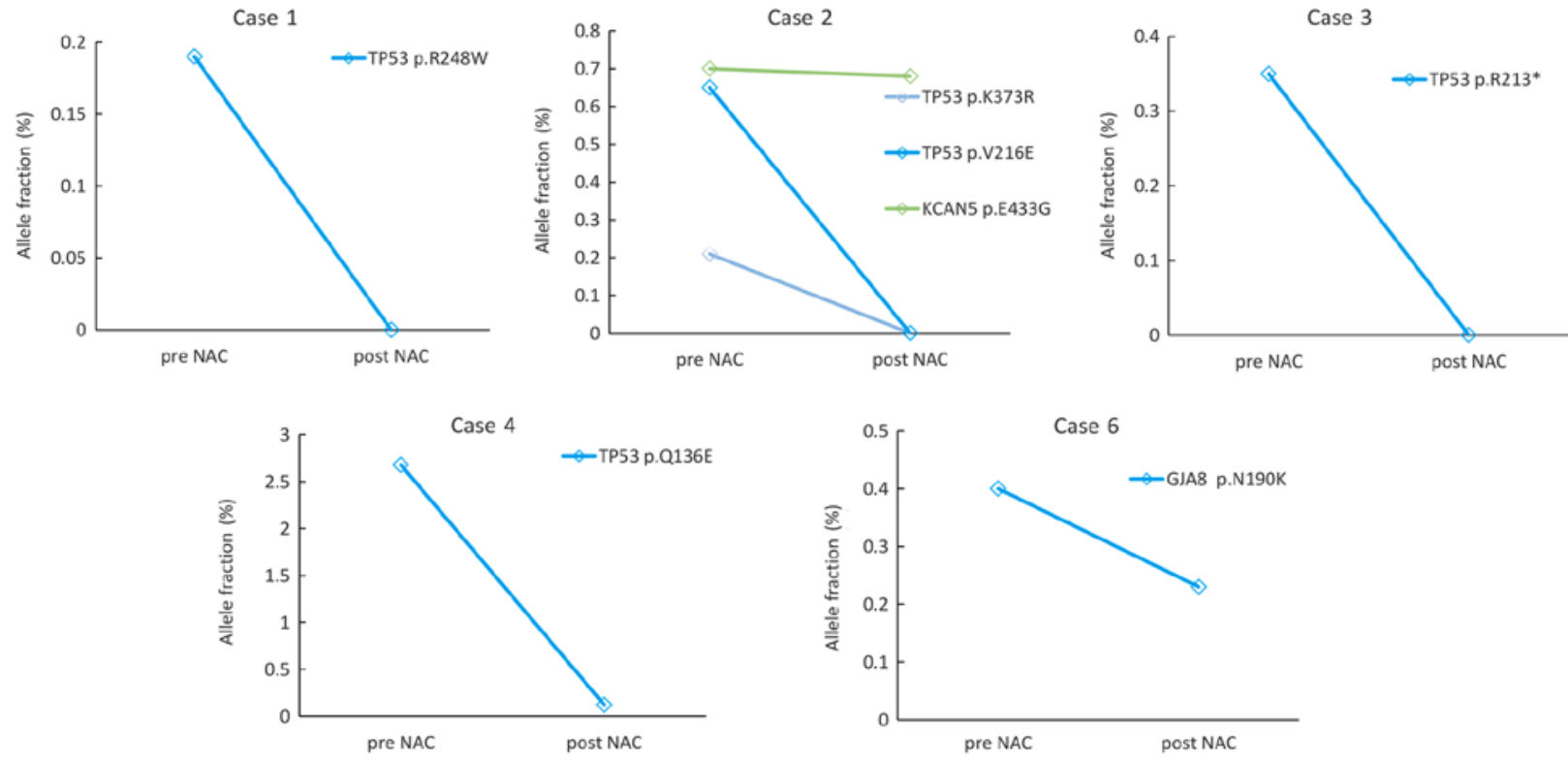

Figure 2. Changes of the allele fractions for somatic mutations detected using CAPP-Seq in NAC-sensitive cases. In Case 1,2,3,4 and 6, the allele frequency of somatic mutations detected by CAPP-seq decreased during treatment. CAPP-Seq, Cancer Personalized Profiling by deep Sequencing; NAC, neoadjuvant chemotherapy.

suggest that minor clones present before the NAC might expand under the selective pressure of chemotherapy.

Association of TP53 VAF with the response to NAC. According to The Cancer Genome Atlas (TCGA), TP53 mutations are found in $94.6 \%$ of patients with high-grade serous ovarian cancer (25). In this study, TP53 mutations were detected at a high frequency. Thus, we compared the changes of the TP53 VAF between the NAC-sensitive and NAC-resistant patient groups. Five and four mutation sites of TP53 were detected in the NAC-sensitive and NAC-resistant cases, respectively (Fig. S2). Change of the TP53 VAF was evaluated as a fold change in $\log$ based 2 , which was the ratio of the post
TP53 to pre TP53 VAF. The TP53 VAF increased significantly during NAC in the NAC-resistant patient group as compared to the NAC-sensitive patient groups (Fig. 4). These results suggest that chemotherapy induces as increase of the mutant allele frequency of TP53 in NAC-resistant cases.

Blood tumor mutation burden (bTMB) and response to chemotherapy. A recent study has shown a significant positive correlation between the tissue-based TMB (tTMB) and bTMB (22), suggesting that the bTMB could serve as a surrogate markers of the TMB. In this study, the bTMB was evaluated as the number of non-synonymous mutation number per $\mathrm{Mb}$, and changes in the bTMB post-NAC relative the level 
Case 7

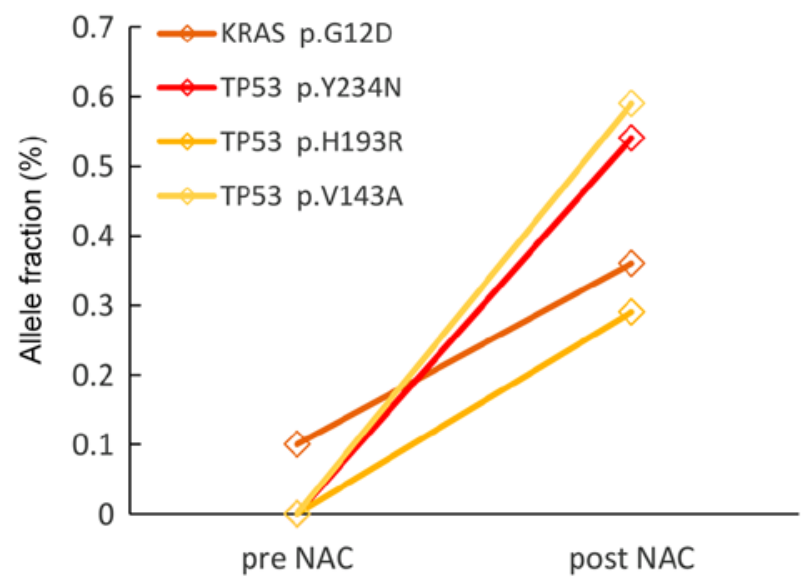

Case 10

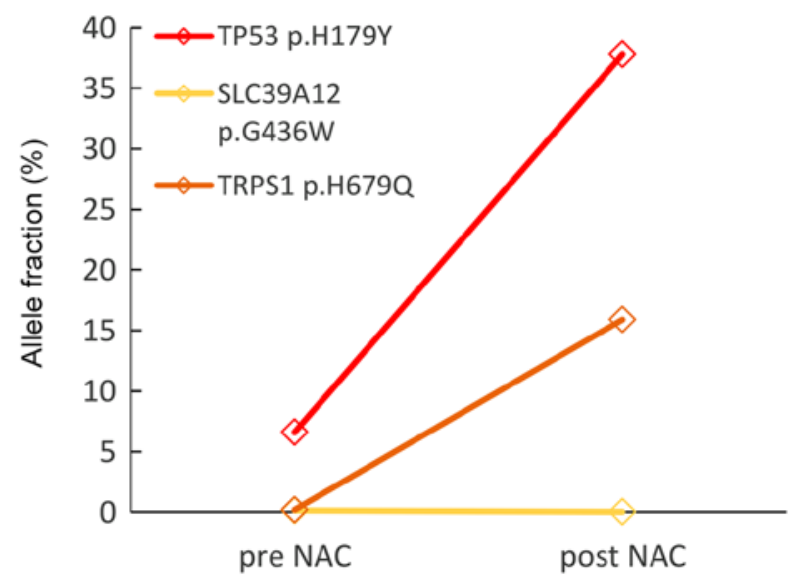

Figure 3. Changes of allele fractions for somatic mutations detected using CAPP-Seq in NAC-resistant cases. In Case 7 and 10 , allele frequency of somatic mutations detected using CAPP-seq (except SLC39A12 p.G436W in Case 10) increased during treatment. CAPP-Seq, Cancer Personalized Profiling by deep Sequencing; NAC, neoadjuvant chemotherapy.

A

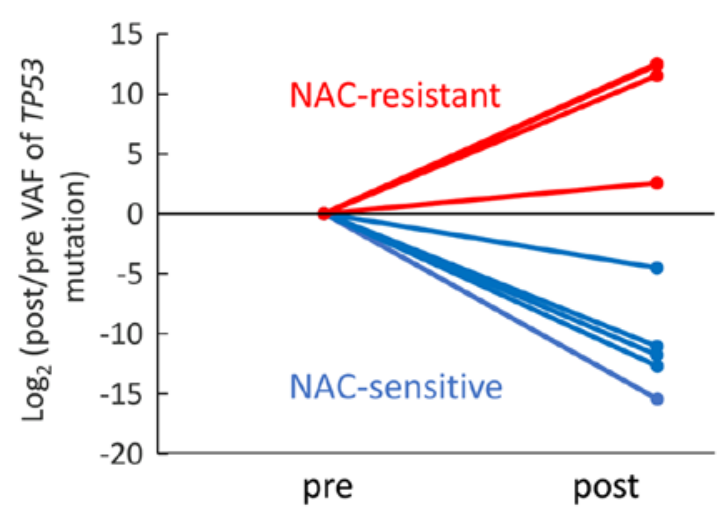

B

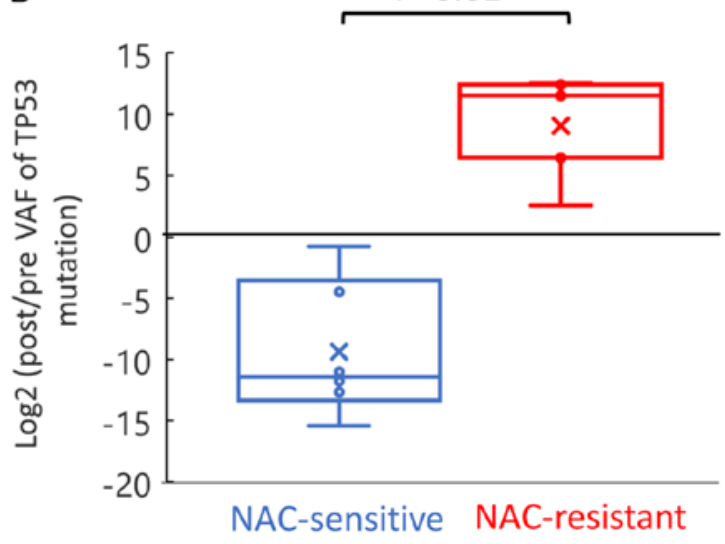

Figure 4. Changes in the VAF of TP53 in the NAC-sensitive and -resistant patient groups. (A) Change of TP53 VAF decreased during treatment in NAC-sensitive groups (blue), whereas the change of TP53 VAF increased during treatment in NAC-resistant groups (red). (B) Significant difference in the change of TP53 VAF was observed between NAC-sensitive (blue) and NAC-resistant (red) patient group $(\mathrm{P}=0.01)$. The statistical analyses were performed using the Mann-Whitney $\mathrm{U}$ test. $\mathrm{P}<0.05$ was considered to indicate a statistically significant difference. VAF, variant allele frequency; NAC, neoadjuvant chemotherapy.

at the baseline were shown to be associated with the response to chemotherapy (Fig. 5). There was no significant difference in the pre-NAC baseline bTMB level between the NAC-sensitive and -resistant patients (15.15/Mb and 10.10/Mb, Fig. 5A). In the NAC-sensitive group, the median values of the bTMB in the pre- and post- NAC samples was $15.15 / \mathrm{Mb}$ and $7.58 / \mathrm{Mb}$, respectively. Thus, the bTMB decreased significantly during the NAC ( $\mathrm{P}=0.049$, Fig. 5B). On the other hand, in the NAC-resistant patient group, a trend towards a light increase of the bTMB value in the post-NAC ctDNA $(15.15 / \mathrm{Mb})$ as compared to the pre-NAC ctDNA was observed $(10.10 / \mathrm{Mb})$, although this difference was not significant (Fig. 5C). These results suggest that the number of mutations detected in ctDNA by CAPP-Seq may decrease as the tumor burden reduce during treatment.

\section{Discussion}

To the best of our knowledge, the current study is the first to demonstrate the usefulness of analyzing comprehensive gene alterations in liquid biopsy-derived ctDNA using CAPP-Seq in advanced ovarian cancer patients receiving NAC. Using CAPP-Seq, we could detect changes in non-synonymous somatic mutations in the plasma ctDNA between NAC-sensitive and NAC-resistant patients, including changes of TP53 VAF and $\mathrm{bTMB}$ during treatment. These findings suggest that CAPP-Seq based molecular profiling of ctDNA may be useful for monitoring the treatment response in advanced ovarian cancer patients receiving NAC.

Recently, some studies have demonstrated the usefulness of detection of multiple somatic mutations by CAPP-seq. The use of targeted hybrid capture with high-throughput sequencing and a specialized bioinformatics workflow technique for plasma ctDNA allows highly sensitive, non-invasive, and low-cost ctDNA detection $(14,15)$. Our previous studies have demonstrated the feasibility of ctDNA gene mutation profiling using CAPP-Seq, not only in cases of gynecological cancer, but also in metastasis from colorectal cancer to the ovary, which exhibited the well-known genetic signature of 
A All cases

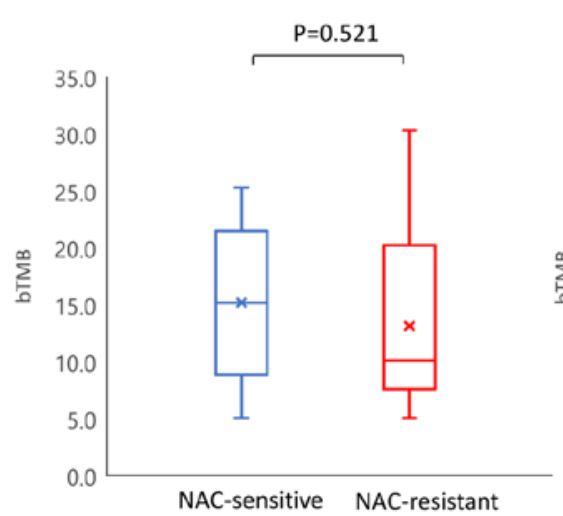

B NAC-sensitive cases

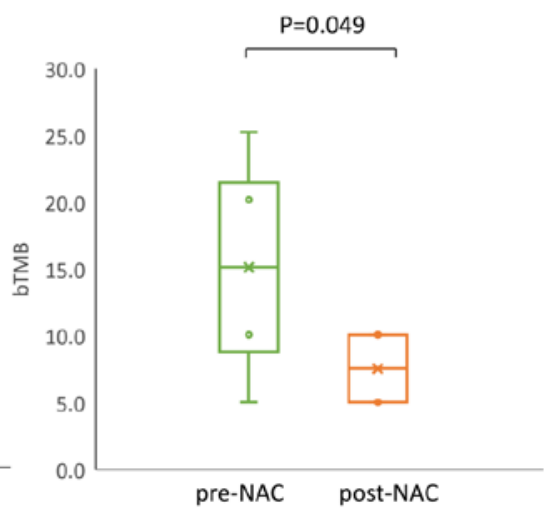

C NAC-resistant cases

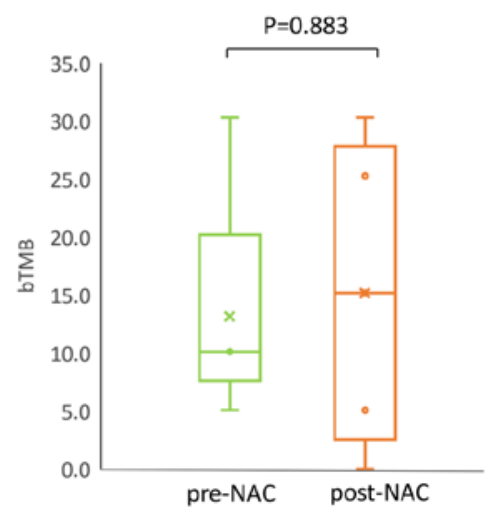

Figure 5. (A) Changes of bTMB in NAC-sensitive and -resistant cases. bTMB at the baseline was calculated using CAPP-seq. No significant difference was observed between NAC-sensitive and -resistant patient groups. (B) Changes in the bTMB post-NAC compared with measured pre-NAC in NAC-sensitive patient groups. (C) Changes in the bTMB post-NAC compared with measured post-NAC in NAC-resistant patient groups. A significant decrease of bTMB during NAC was observed in NAC-sensitive patient groups $(\mathrm{P}<0.05)$. Statistical analyses were performed using the Mann-Whitney $\mathrm{U}$ test. $\mathrm{P}<0.05$ was considered to indicate a statistically significant difference. bTMB, blood tumor mutation burden; NAC, neoadjuvant chemotherapy; CAPP-Seq, Cancer Personalized Profiling by deep Sequencing.

colorectal cancer (KRAS, APC and TP53 mutations and MET copy-number gain) $(18,19)$.

Previous reports showed the usefulness of the plasma ctDNA concentration as a diagnostic, prognostic and predictive biomarker in patients with ovarian cancer (26-30). However, the clinical usefulness of this parameter is still controversial because of the different assay methods, such as real-time PCR and fluorescence staining, and different sample sources such as plasma or serum. A recent meta-analysis reported that quantitative analysis of ctDNA was associated with low sensitivity, 0.70 (95\% CI 0.65-0.74), but high specificity, 0.90 (95\% CI 0.87-0.93) for the diagnosis of ovarian cancer (30). Capizzi et al showed that the ctDNA concentration in patients with ovarian cancer measured before chemotherapy decreased significantly after chemotherapy (26). In our study, there was no significant difference in the overall ctDNA concentration during NAC treatment between the NAC-sensitive and NAC-resistant cases, although the sample size was limited. On the other hand, a recent review reported that independent genetic alterations in the ctDNA showed both higher sensitivity and specificity as compared to the overall ctDNA concentration (31). Some studies have focused on using TP53 mutations in the ctDNA as the most common somatic mutation in ovarian cancer. Swisher et al showed that the presence of TP53 mutation in the ctDNA of ovarian cancer patients was an independent predictor of decreased survival (32). Other studies showed that undetectable TP53 mutations or reduction in TP53 mutations after chemotherapy in ovarian cancer patients was significantly associated with a better prognosis $(33,34)$. In our study, there were six sites of TP53 mutations detected by CAPP-seq. We demonstrated a significantly higher degree of change of TP53 mutations in the NAC-resistant cases. Based on this result, we speculated that chemotherapy may induce an increase in the mutant allele frequency of TP53 in NAC-resistant cases.

In our study, CAPP-Seq enabled sensitive detection of multiple classes of somatic mutations and deep sequencing. Indeed, LRRTM1, BRINP2, CDH9 and GRM1 were detected only after NAC in the NAC-resistant cases. It is suspected that minor clones with these mutations before NAC might become major clones during chemotherapy. Although there have been no previous reports of these mutations in advanced ovarian cancer, these mutations might be related to resistance to chemotherapy. We also found EFGR amplification in post-NAC samples of NAC-resistant cases (Patient no. 8 and 10). Some previous studies reported that EGFR amplification was significantly associated with a shorter overall survival in ovarian cancer patients $(35,36)$. Lassus et al reported that EGFR amplification and overexpression in serous ovarian cancers were associated with a shorter overall and disease-free survival (36). EGFR amplification detected in the ctDNA might be a useful biomarker of the response to chemotherapy in patients with ovarian cancer.

The detailed mechanism of changes in the gene mutation profiles of ctDNA in each NAC-sensitive or NAC-resistant patient shown in our study remains to be clarified. We speculated that one reason for the phenomenon might be due to increasing or decreasing tumor burden with response to chemotherapy. As another mechanism, considering that ovarian cancer is likely to show tumor heterogeneity, our data may reflect that NAC could induce clonal evolution and might lead to appearance of chemotherapy-resistant clones.

The present study demonstrated, for the first time, changes in the bTMB in NAC -sensitive and NAC-resistant ovarian cancer patients. The bTMB decreased significantly during treatment in the NAC-sensitive cases, while no significant change of the bTMB was observed in the NAC-resistant cases. However, the baseline bTMB level measured before the NAC was not a predictor of the response to chemotherapy within the small number of patients entered in this study. In patients with non-small cell lung cancer, the high bTMB ( $\geqq 16 \mathrm{Mb}$ ) is reported to be associated with better response to anti-PD-L1/PD-1 therapies (22). High-tissue TMB was shown to be associated with longer survival in patients with high-grade serous ovarian cancer carrying BRCA1 or BRCA2 mutations (23). However, no reports have been published between bTMB and chemosensitivity of at least ovarian cancers, and the mechanism of the association 
of blood-based TMB with efficacy of chemotherapy remains unclear in ovarian cancer. Further study should be conducted whether bTMB, like tissue-TMB, may be a predictive biomarker of response to chemotherapy or immune checkpoint inhibitors for stratification of individual therapeutic strategies in patients with advanced ovarian cancer.

There were several limitations of our study. First, this was a retrospective study with a small sample size. Second, we did not conduct the tumor tissue-derived DNA sequencing using CAPP-Seq. CAPP-Seq is currently specialized for plasma ctDNA, and therefore it remains a future challenge to expand its application to formalin-fixed paraffin-embedded tumor tissue. Further experiments using tumor DNA are needed to explore the ability/sensitivity of detection of tumor mutations in ctDNA. Third, blood samples were collected only at two points, pre- and post-NAC in this study. If blood samples were collected at a greater number of time points during chemotherapy, changes in the allele frequency can also be detected in detail. Finally, approach to analyze ctDNA remains technically challenge in order to distinguish strictly ctDNA from cell-free DNA derived from normal tissues and blood cells. CAPP-seq is a novel NGS-based ultrasensitive method for ctDNA analysis and several studies showed that the somatic mutations detected by CAPP-seq using the optimal settings of cut-off values could reflect tumor-specific mutations in ctDNA $(14,15,18-20)$.

In conclusion, we demonstrated that molecular profiling and monitoring of gene mutations could be successfully performed by CAPP-Seq in advanced ovarian cancer patients receiving NAC. Furthermore, we suggest that the TP53 mutation and bTMB detected by liquid biopsy may be new biomarkers of the response to NAC. These findings shown by liquid biopsy might enable detection and assessment of post-treatment minimal residual disease (MRD). Although extensive research has been conducted on the genetic profiling of gynecological cancers so far conducted using tumor tissue-derived DNA (25), tumor biopsy or surgical resection for sufficient samples is often difficult particularly in case of advanced/recurrent ovarian cancer. Liquid biopsy is minimally invasive, allows easily serial measurements and may also allow tumor heterogeneity to be represented at real-time point. Genetic profiling of ctDNA in gynecological cancers by CAPP-Seq may help in the establishment of more efficient personalized therapeutic algorithms and real-time therapy monitoring.

\section{Acknowledgements}

Not applicable.

\section{Funding}

This work was supported by Ministry of Education, Culture, Sports, Science and Technology of Japan Grants-in-Aid for Scientific Research Grant (grant no. JP19K18677).

\section{Availability of data and materials}

The datasets obtained and/or analyzed during the current study are available from the corresponding author on reasonable request.

\section{Author contribution}

TN and NI performed the experiments, interpreted the data and wrote the manuscript. KS and TN analyzed the circulating tumor DNA sequencing and interpreted the data. KM, HM, TY and ST collected and prepared blood samples. KS contributed reagents, materials and analytical tools and supervised the entire project. $\mathrm{KN}$ and $\mathrm{KI}$ designed the research, interpreted the data and wrote the paper. All authors reviewed the results and approved the final version of the manuscript.

\section{Ethics approval and consent to participate}

This study was approved by the ethics committee of the Institutional Review Board of Wakayama Medical University Faculty of Medicine (authorization no. 2025) and Kindai University Faculty of Medicine (authorization no. 29-066). All patients in the current study provided written informed consent for the use of their plasma and tissue samples.

\section{Patient consent for publication}

Written informed consent for publication of the present report was obtained from the patient.

\section{Competing interests}

The authors declare that they have no competing interests.

\section{References}

1. Siegel R, Naishadham D and Jemal A: Cancer statistics, 2013. CA Cancer J Clin 63: 11-30, 2013.

2. Chi DS, Eisenhauer EL,Zivanovic O, Sonoda Y, Abu-Rustum NR, Levine DA, Guile MW, Bristow RE, Aghajanian C and Barakat RR: Improved progression-free and overall survival in advanced ovarian cancer as a result of a change in surgical paradigm. Gynecol Oncol 114: 26-31, 2009.

3. Fagotti A, Ferrandina G, Vizzielli G, Fanfani F, Gallotta V, Chiantera V Costantini B, Margariti PA, Gueli Alletti S, Cosentino F, et al: Phase III randomised clinical trial comparing primary surgery versus neoadjuvant chemotherapy in advanced epithelial ovarian cancer with high tumour load (SCORPION trial): Final analysis of peri-operative outcome. Eur J Cancer 59: 22-33, 2016.

4. Kehoe S, Hook J, Nankivell M, Jayson GC, Kitchener H, Lopes T, Luesley D, Perren T, Bannoo S, Mascarenhas M, et al: Primary chemotherapy versus primary surgery for newly diagnosed advanced ovarian cancer (CHORUS): An open-label, randomised, controlled, non-inferiority trial. Lancet 386: 249-257, 2015.

5. Vergote I, Tropé CG, Amant F, Kristensen GB, Ehlen T, Johnson N, Verheijen RH, van der Burg ME, Lacave AJ, Panici PB, et al: Neoadjuvant chemotherapy or primary surgery in stage IIIC or IV ovarian cancer. N Engl J Med 363: 943-953, 2010.

6. Pelissier A, Bonneau C, Chéreau E, de La Motte Rouge T, Fourchotte V, Daraï E and Rouzier R: CA125 kinetic parameters predict optimal cytoreduction in patients with advanced epithelial ovarian cancer treated with neoadjuvant chemotherapy. Gynecol Oncol 135: 542-546, 2014.

7. Rodriguez N, Rauh-Hain JA, Shoni M, Berkowitz RS, Muto MG, Feltmate C, Schorge JO, Del Carmen MG, Matulonis UA and Horowitz NS: Changes in serum CA-125 can predict optimal cytoreduction to no gross residual disease in patients with advanced stage ovarian cancer treated with neoadjuvant chemotherapy. Gynecol Oncol 125: 362-366, 2012. 
8. Zeng J, Yin J, Song X, Jin Y, Li Y and Pan L: Reduction of CA125 levels during neoadjuvant chemotherapy can predict cytoreduction to no visible residual disease in patients with advanced epithelial ovarian cancer, primary carcinoma of fallopian tube and peritoneal carcinoma. J Cancer 7: 2327-2332, 2016.

9. Diaz LA Jr and Bardelli A: Liquid biopsies: Genotyping circulating tumor DNA. J Clin Oncol 32: 579-586, 2014.

10. Diehl F, Schmidt K, Choti MA, Romans K, Goodman S, Li M, Thornton K, Agrawal N, Sokoll L, Szabo SA, et al: Circulating mutant DNA to assess tumor dynamics. Nat Med 14: 985-990, 2008.

11. Vogelstein B and Kinzler KW: Digital PCR. Proc Natl Acad Sci USA 96: 9236-9241, 1999.

12. Bettegowda C, Sausen M, Leary RJ, Kinde I, Wang Y Agrawal N, Bartlett BR, Wang H, Luber B, Alani RM, et al: Detection of circulating tumor DNA in early- and late-stage human malignancies. Sci Transl Med 6: 224ra24, 2014.

13. Narayan A, Carriero NJ, Gettinger SN, Kluytenaar J, Kozak KR, Yock TI, Muscato NE, Ugarelli P, Decker RH and Patel AA Ultrasensitive measurement of hotspot mutations in tumor DNA in blood using error-suppressed multiplexed deep sequencing. Cancer Res 72: 3492-3498, 2012.

14. Newman AM, Bratman SV, To J, Wynne JF, Eclov NC, Modlin LA, Liu CL, Neal JW, Wakelee HA, Merritt RE, et al: An ultrasensitive method for quantitating circulating tumor DNA with broad patient coverage. Nat Med 20: 548-554, 2014

15. Newman AM, Lovejoy AF, Klass DM, Kurtz DM, Chabon JJ, Scherer F, Stehr H, Liu CL, Bratman SV, Say C, et al: Integrated digital error suppression for improved detection of circulating tumor DNA. Nat Biotechnol 34: 547-555, 2016.

16. Scherer F, Kurtz DM, Newman AM, Stehr H, Craig AF, Esfahani MS, Lovejoy AF, Chabon JJ, Klass DM, Liu CL, et al: Distinct biological subtypes and patterns of genome evolution in lymphoma revealed by circulating tumor DNA. Sci Transl Med 8: 364ra155, 2016.

17. Przybyl J, Chabon JJ, Spans L, Ganjoo KN, Vennam S, Newman AM, Forgó E, Varma S, Zhu S, Debiec-Rychter M, et al: Combination approach for detecting different types of alterations in circulating tumor DNA in leiomyosarcoma. Clin Cancer Res 24: 2688-2699, 2018.

18. Iwahashi N, Sakai K, Noguchi T, Yahata T, Toujima S, Nishio K and Ino K: A comprehensive gene mutation analysis of liquid biopsy samples from patients with metastatic colorectal cancer to the ovary: A case report. Oncol Lett 16: 6431-6436, 2018.

19. Iwahashi N, Sakai K, Noguchi T, Yahata T, Matsukawa H, Toujima S, Nishio K and Ino K: Liquid biopsy-based comprehensive gene mutation profiling for gynecological cancer using cancer personalized profiling by deep sequencing. Sci Rep 9 . 10426, 2019

20. Otsubo K, Sakai K, Takeshita M, Harada D, Azuma K, Ota K, Akamatsu H, Goto K, Horiike A, Kurata T, et al: Genetic profiling of non-small cell lung cancer at development of resistance to first- or second-generation EGFR-TKIs by CAPP-Seq analysis of circulating tumor DNA. Oncologist 24: 1022-1026, 2019.

21. Rizvi H, Sanchez-Vega F, La K, Chatila W, Jonsson P, Halpenny D, Plodkowski A, Long N, Sauter JL, Rekhtman N, et al: Molecular determinants of response to anti-programmed cell death (PD)-1 and anti-programmed death-ligand 1 (PD-L1) blockade in patients with non-small-cell lung cancer profiled with targeted next-generation sequencing. J Clin Oncol 36: 633-641, 2018.

22. Gandara DR, Paul SM, Kowanetz M, Schleifman E, Zou W, Li Y, Rittmeyer A, Fehrenbacher L, Otto G, Malboeuf C, et al: Blood-based tumor mutational burden as a predictor of clinical benefit in non-small-cell lung cancer patients treated with atezolizumab. Nat Med 24: 1441-1448, 2018.
23. Birkbak NJ, Kochupurakkal B, Izarzugaza JM, Eklund AC, Li Y, Liu J, Szallasi Z, Matulonis UA, Richardson AL, Iglehart JD and Wang ZC: Tumor mutation burden forecasts outcome in ovarian cancer with BRCA1 or BRCA2 mutations. PLoS One 8: e80023, 2013.

24. Narahara M, Higasa K, Nakamura S, Tabara Y, Kawaguchi T, Ishii M, Matsubara K, Matsuda F and Yamada R: Large-scale East-Asian eQTL mapping reveals novel candidate genes for LD mapping and the genomic landscape of transcriptional effects of sequence variants. PLoS One 9: e100924, 2014.

25. Cancer Genome Atlas Research Network: Integrated genomic analyses of ovarian carcinoma. Nature 474: 609-615, 2011.

26. Capizzi E, Gabusi E, Grigioni AD, De Iaco P, Rosati M, Zamagni C and Fiorentino M: Quantification of free plasma DNA before and after chemotherapy in patients with advanced epithelial ovarian cancer. Diagn Mol Pathol 17: 34-38, 2008.

27. Gu XH, Wu NW and Liu XS: Application of serum DNA quantification in the diagnosis of epithelial ovarian cancer. Matern Child Health Care China 24: 1413-1415, 2009.

28. Holdenrieder S, Stieber P, Bodenmuller H, Busch M, Fertig G, Fürst H, Schalhorn A, Schmeller N, Untch M and Seidel D: Nucleosomes in serum of patients with benign and malignant diseases. Int J Cancer 95: 114-120, 2001.

29. Kamat AA, Baldwin M, Urbauer D, Dang D, Han LY, Godwin A, Karlan BY, Simpson JL, Gershenson DM, Coleman RL, et al: Plasma cell-free DNA in ovarian cancer: An independent prognostic biomarker. Cancer 116: 1918-1925, 2010.

30. Zhou Q, Li W, Leng B, Zheng W, He Z, Zuo M and Chen A: Circulating cell Free DNA as the diagnostic marker for ovarian cancer: A systematic review and meta-analysis. PLoS One 11: e0155495, 2016.

31. Barbosa A, Peixoto A, Pinto P, Pinheiro M and Teixeira MR: Potential clinical applications of circulating cell-free DNA in ovarian cancer patients. Expert Rev Mol Med 20: e6, 2018.

32. Swisher EM, Wollan M, Mahtani SM, Willner JB, Garcia R, Goff BA and King MC: Tumor-specific p53 sequences in blood and peritoneal fluid of women with epithelial ovarian cancer. Am J Obstet Gynecol 193: 662-667, 2005.

33. Kim YM, Lee SW, Lee YJ, Lee HY, Lee JE and Choi EK: Prospective study of the efficacy and utility of TP53 mutations in circulating tumor DNA as a non-invasive biomarker of treatment response monitoring in patients with high-grade serous ovarian carcinoma. J Gynecol Oncol 30: e32, 2019.

34. Pereira E, Camacho-Vanegas O, Anand S, Sebra R, Catalina Camacho S, Garnar-Wortzel L, Nair N, Moshier E, Wooten M, Uzilov A, et al: Personalized circulating tumor DNA biomarkers dynamically predict treatment response and survival in gynecologic cancers. PLoS One 10: e 0145754, 2015.

35. Lafky JM, Wilken JA, Baron AT and Maihle NJ: Clinical implications of the ErbB/epidermal growth factor (EGF) receptor family and its ligands in ovarian cancer. Biochim Biophys Acta 1785: 232-265, 2008.

36. Lassus H, Sihto H, Leminen A, Joensuu H, Isola J, Nupponen NN and Butzow R: Gene amplification, mutation, and protein expression of EGFR and mutations of ERBB2 in serous ovarian carcinoma. J Mol Med (Berl) 84: 671-681, 2006.

This work is licensed under a Creative Commons Attribution-NonCommercial-NoDerivatives 4.0 International (CC BY-NC-ND 4.0) License. 